\title{
Evaluation of the Neurotrophic Factors in Animal Model of Myelin Destruction Induced by Cuprizone in C57bl/6 Mice
}

\author{
Arefeh Shirazi ${ }^{1}$, Fereshteh Golab ${ }^{2 *}$, Nima Sanadgol ${ }^{3}$, Mahmood Barati ${ }^{4}$, Reza Mohammad Salehi ${ }^{5}$, Gelareh \\ Vahabzadeh $^{6}$, Zeinab Shadalui' ${ }^{2}$, Saeed Rezaei Zarchi ${ }^{1}$
}

${ }^{1}$ Biology Department, Payame Noor University, Tehran, Iran

${ }^{2}$ Cellular and Molecular Research Center, Iran University of Medical Sciences, Tehran, Iran

${ }^{3}$ Department of Biology, Faculty of Sciences, University of Zabol, Zabol, Iran

${ }^{4}$ Department of Pharmaceutical Biotechnology, School of Pharmacy, Shahid Beheshti University of Medical Sciences, Tehran, Iran

${ }^{5}$ Molecular Microbiology Research Center, Shahed University, Tehran, Iran

${ }^{6}$ Department of Pharmacology, School of Medicine, Iran University of Medical Sciences, Tehran, Iran

\section{A BSTRACT}

Introduction: Multiple sclerosis (MS) is a chronic inflammatory demyelinating disease of the central nervous system (CNS) with unknown etiology. Neurotrophins are polypeptides belonging to the neurotrophic factor family. Neurotrophins mediate cell survival and proliferation in the nervous system. In this study, we determined the production of various neurotrophins, including brainderived neurotrophic factor (BDNF), ciliary neurotrophic factor (CNTF), and glial cell-derived neurotrophic factor (GDNF) in Cuprizone model of demyelination. Materials and Methods: In order to induce demyelination, animals were treated by Cuprizone. The mice were divided into three groups. The first group was treated by Cuprizone for 5 weeks. The second group was treated by Cuprizone for 5 weeks and normal diet for 1 week. The third (control) group received normal diet for 6 weeks. After the mice were sacrificed, cerebral corpus callosum was removed and evaluated for expression of neurotrophic factors by real time PCR and histological evaluation. Results: After five weeks, we detected a significant increase of BDNF and GDNF compared to the control group. No changes were observed in CNTF expression. After six weeks, expression of BDNF and GDNF were decreased but they had still higher levels compared to control group.

Conclusion: This study suggests that neurotrophins may play a role in pathogenesis of MS.

\section{Key words:}

1. Multiple Sclerosis

2. Nerve Growth Factors

3. Cuprizone

*Corresponding Author: Fereshteh Golab

E-mail:frgol@yahoo.com 


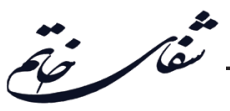

\section{بررسى فاكتورهاى نوروتروفيك در مدل حيوانى تخريب ميلين القاء شده با كويريزون در موشهاى} C57bl/6

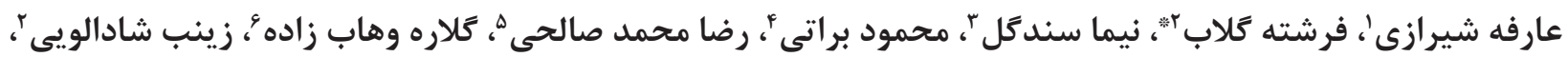
سعيد رضايى زارجى'

'كروه زيستشناسى، دانشكاه بيام نور، تهران، ايران

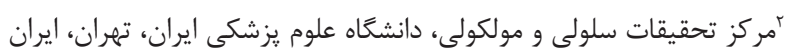

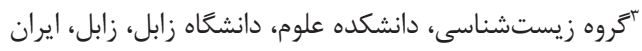

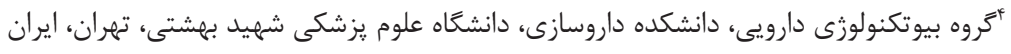

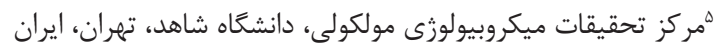

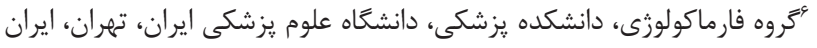

كليد وازهها:

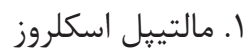

r. باكتورهاى رشد عصبى

"ا. كويريزون

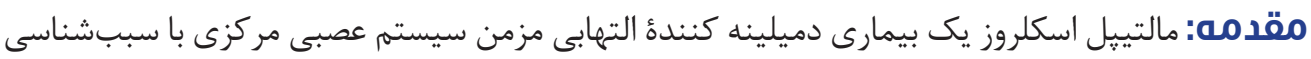

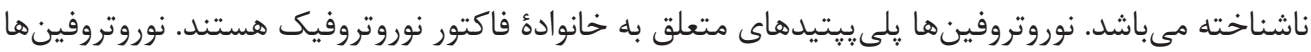

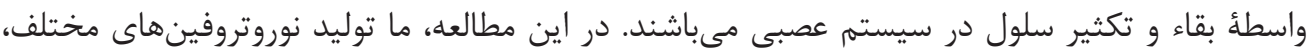

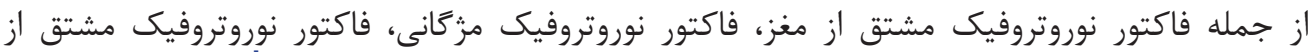

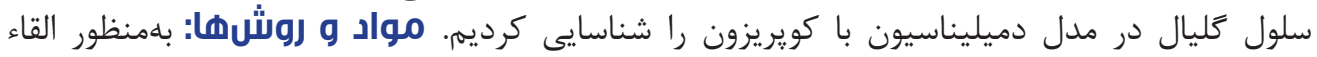

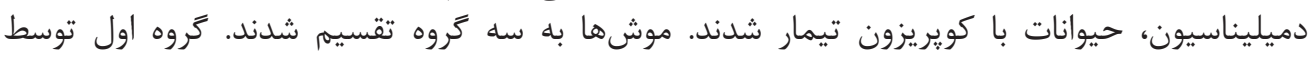

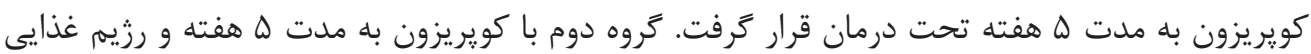

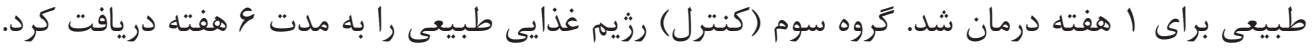

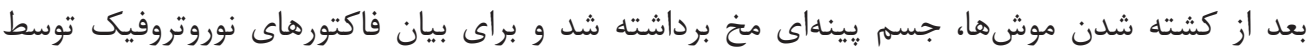
Real Time PCR

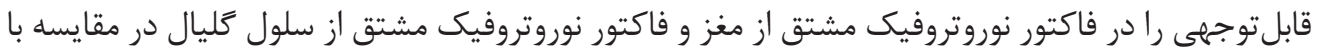

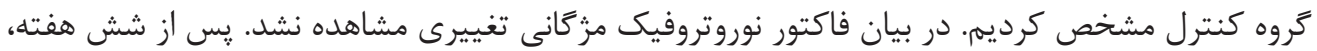

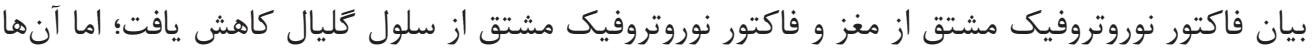

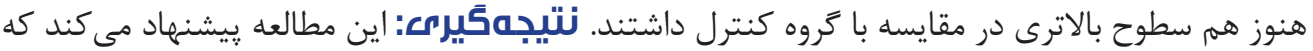

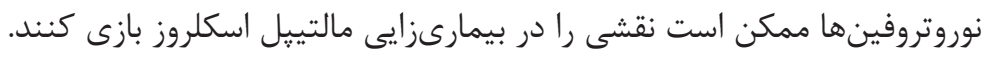

"نويسنده مسئول: فرشته كلاب آدرس الكترونيكى: frgol@yahoo.com 


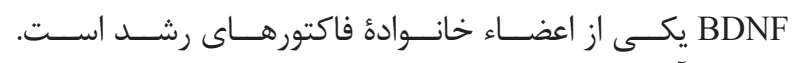

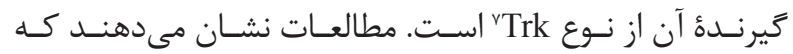

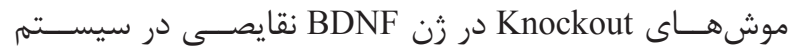

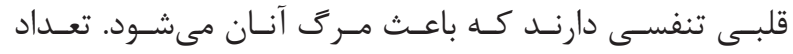

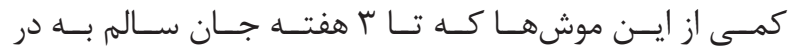

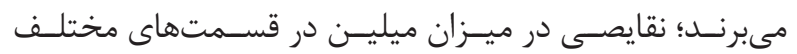

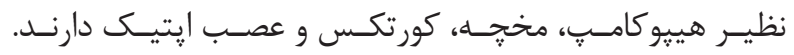

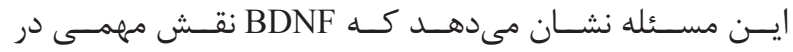

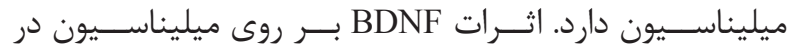

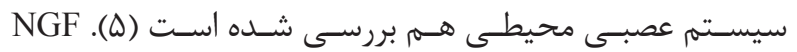

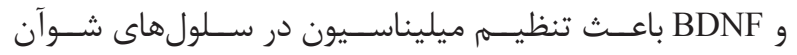

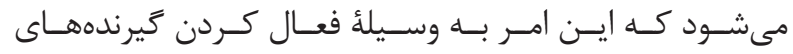

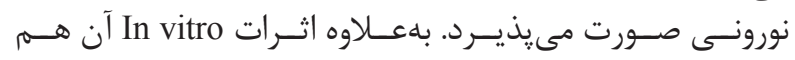

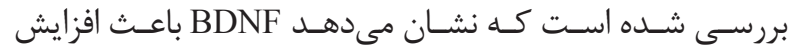

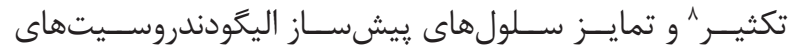

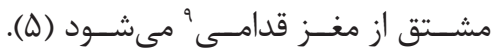

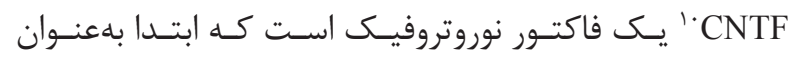

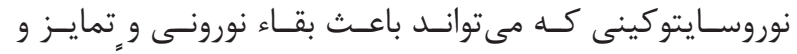

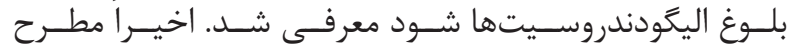

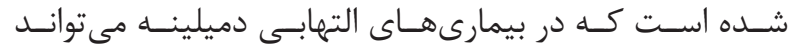

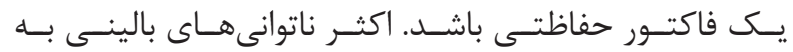

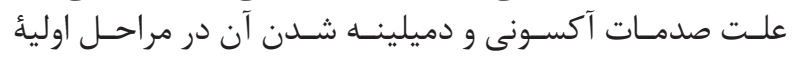

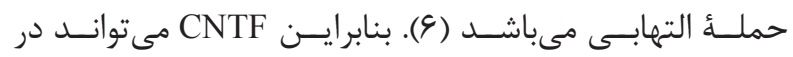

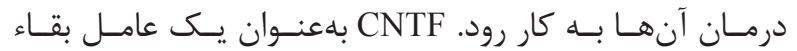

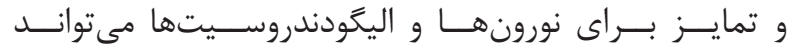

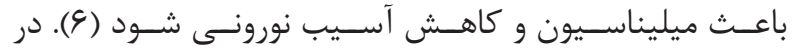

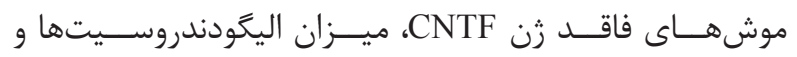

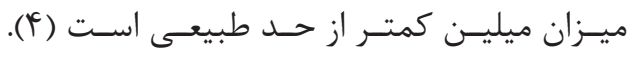

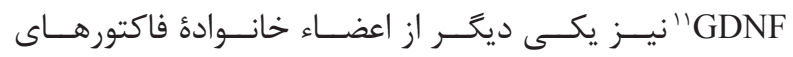

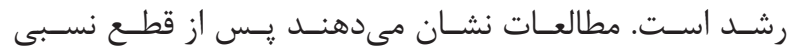

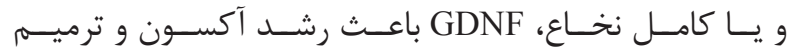

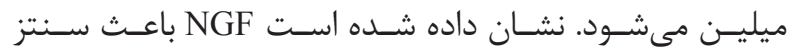

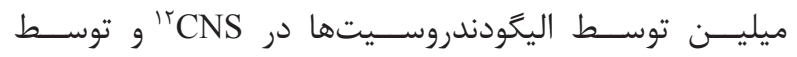

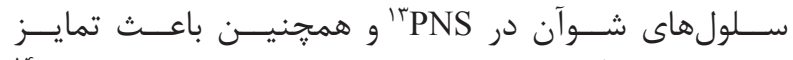

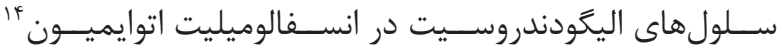

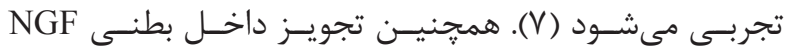

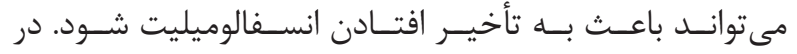

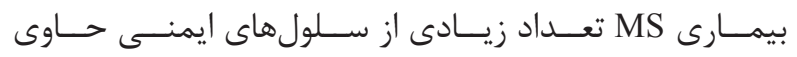

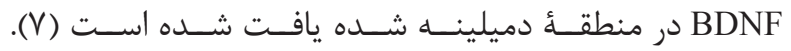

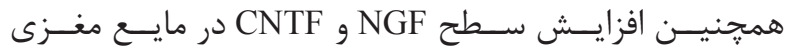

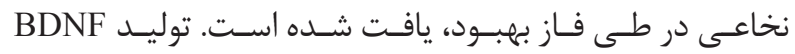

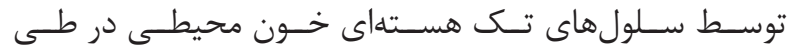

\footnotetext{
${ }^{1}$ Multiple sclerosis

${ }^{2}$ Relapsing-remitting

${ }^{3}$ Nerve growth factor

${ }^{4}$ Brain derived neurotrophic factor

${ }^{5}$ Leukemia Inhibitory Factor

${ }^{6}$ Neurotrophin-3

${ }^{7}$ Tyrosine receptor kinase
}

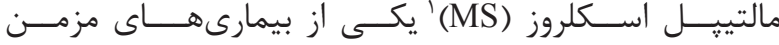

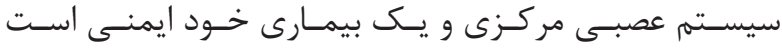

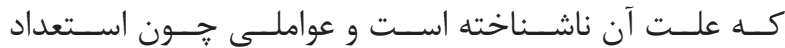

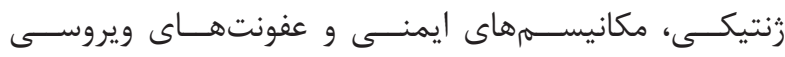

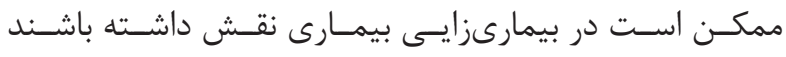

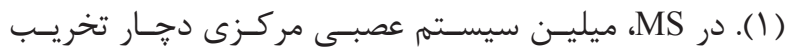

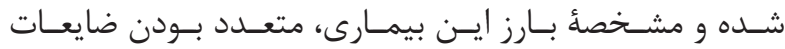

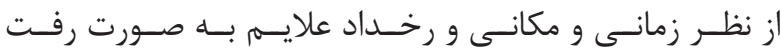

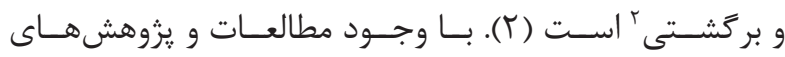

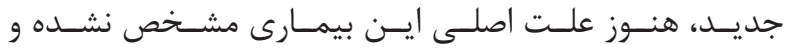

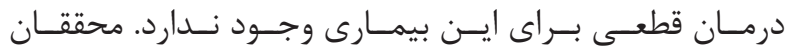

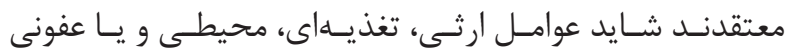

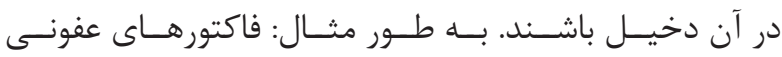

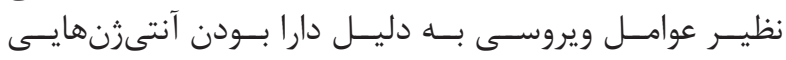

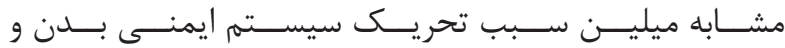

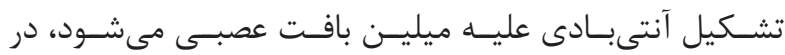

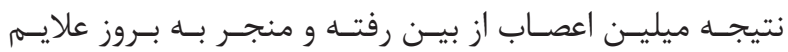

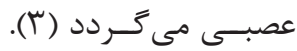

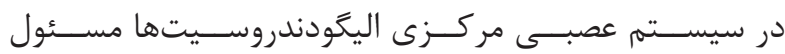

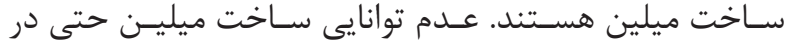

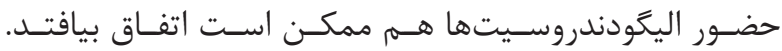

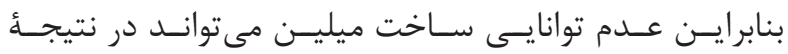

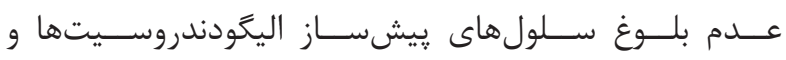

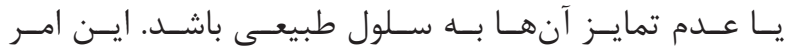

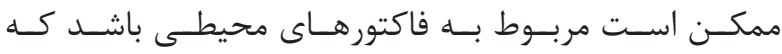

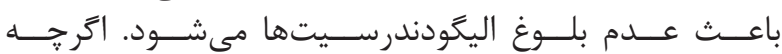

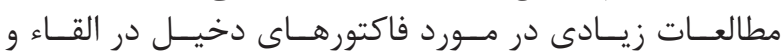

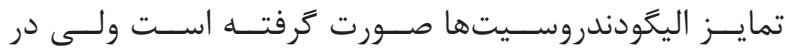

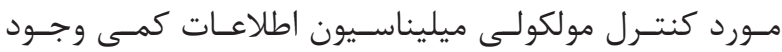

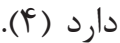

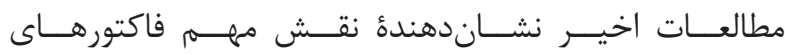

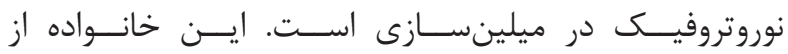

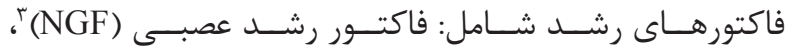

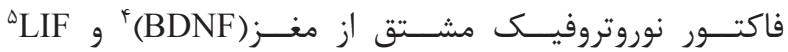

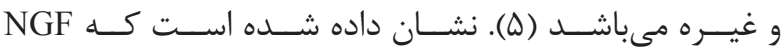

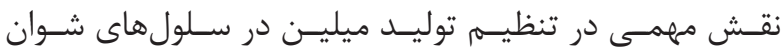

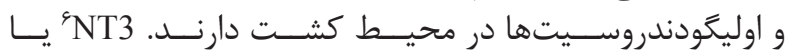

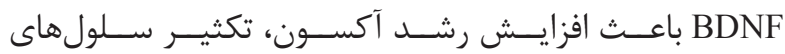

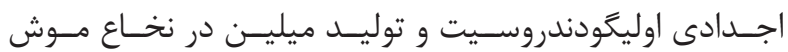

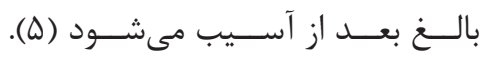

\footnotetext{
${ }^{8}$ Proliferation

${ }^{9}$ Forebrain

${ }^{10}$ Ciliary neurotrophic factor

${ }^{11}$ Glial cell-derived neurotrophic factor

${ }^{12}$ Central nervous system

${ }^{13}$ Peripheral nervous system

${ }^{14}$ Autoimmune encephalomyelitis
} 
كروهبندى حيوانات

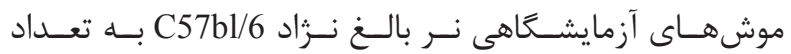

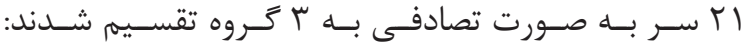

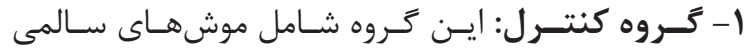

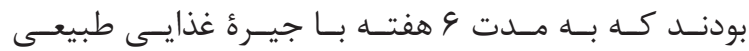

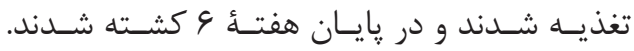

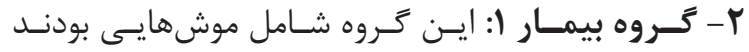

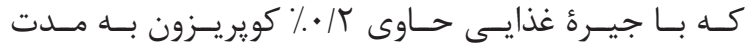

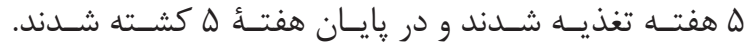

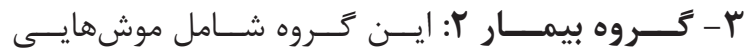

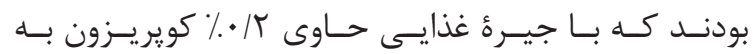

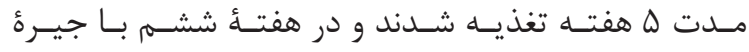

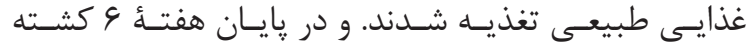

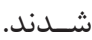

Real Time PCR آمادهسازى نمونه ها براى

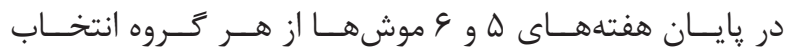

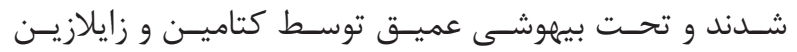

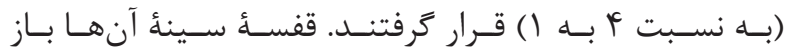

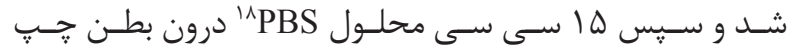

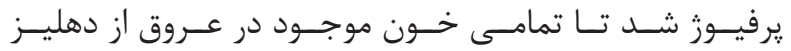

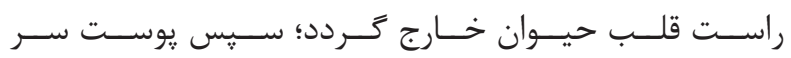

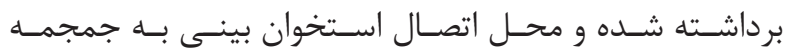

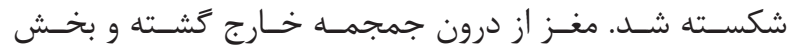

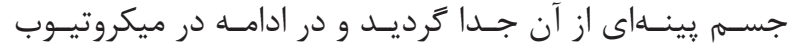

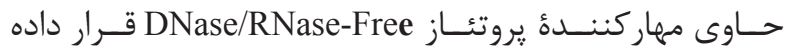

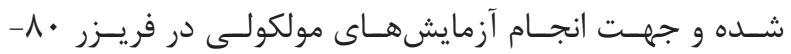

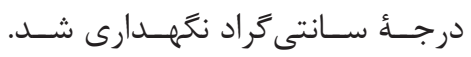

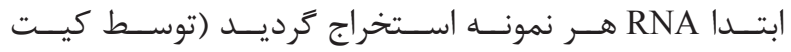
Bioneer

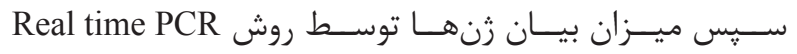

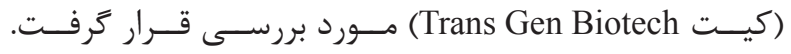

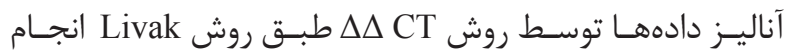

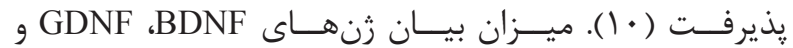

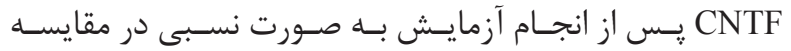

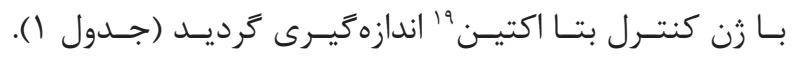

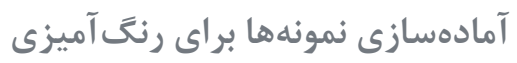

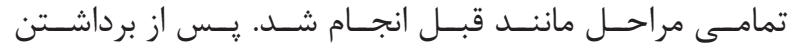

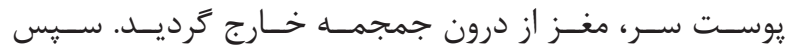

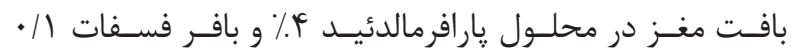

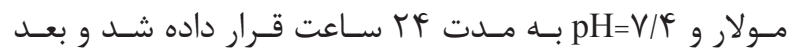

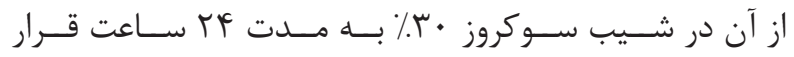

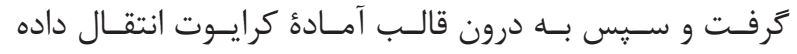

${ }^{15}$ Lysolecithin

${ }^{16}$ Cuprizone

${ }^{17}$ Cerebral corpus callosum
فــاز بهبـود نسـبت بــه فـاز اوليـهـ در بيمـارى MS بيشـــر

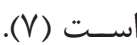

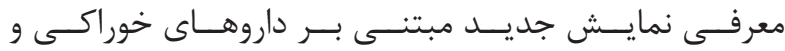

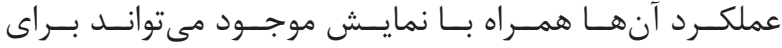

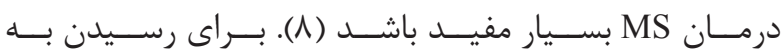

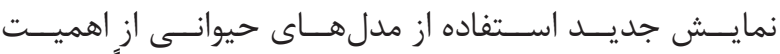

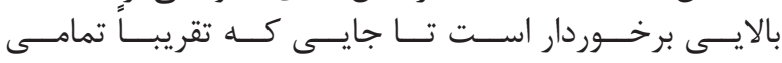

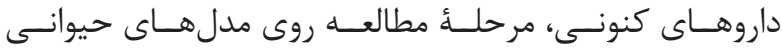

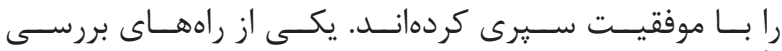

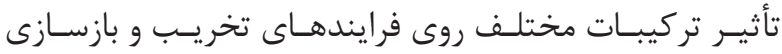

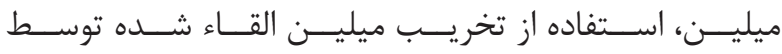

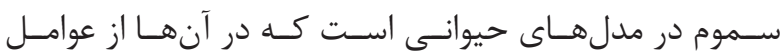

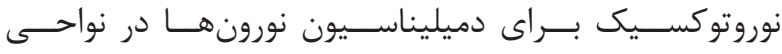

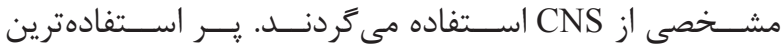

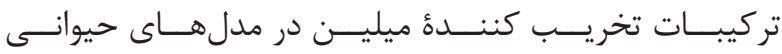

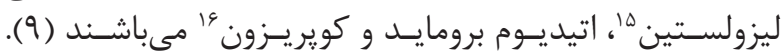

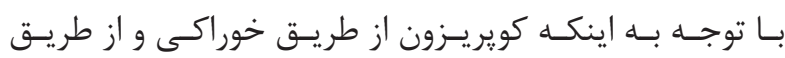

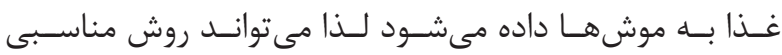

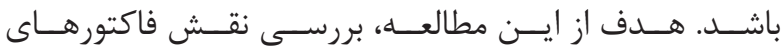

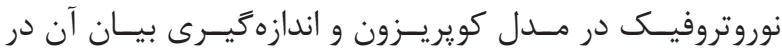

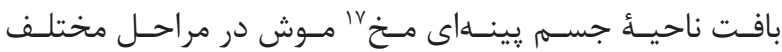

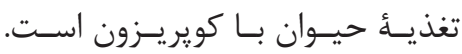

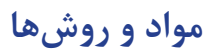

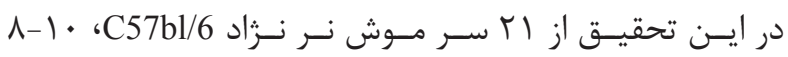

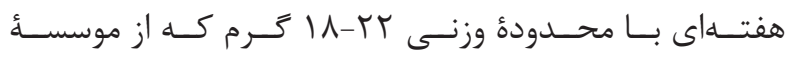

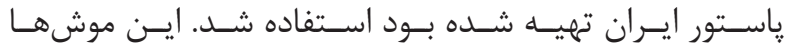

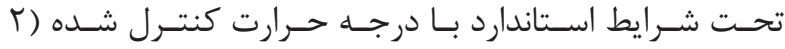

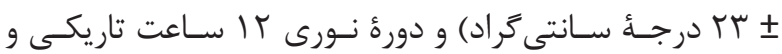

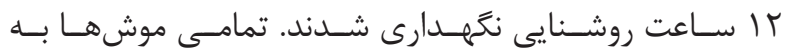

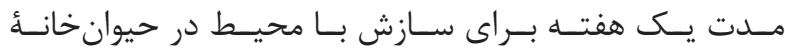

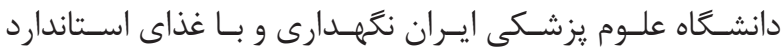
بــه صـورت يـودر شــده تغذيـهـ شـدند.

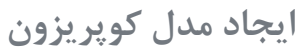

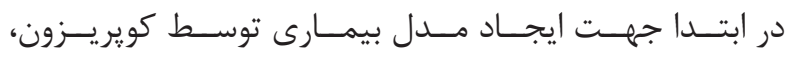

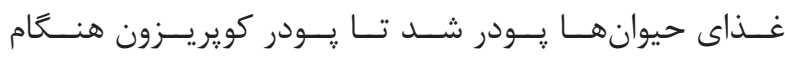

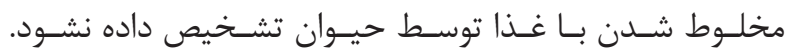

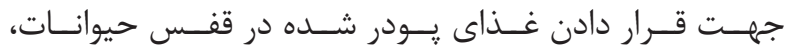

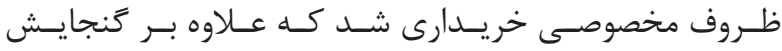

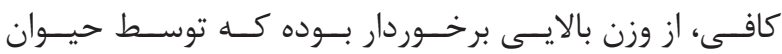

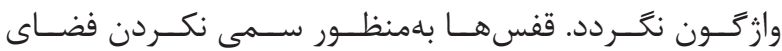

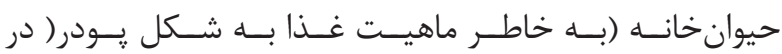

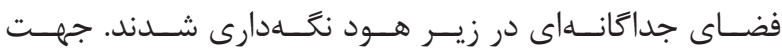

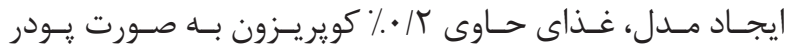

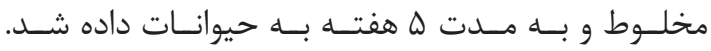

${ }^{18}$ Phosphate buffered saline

${ }^{19} \beta$ Actin 
جدول ا- ترادف يرايمرها و درجة دماى آنلينگ آنها.

\begin{tabular}{|c|c|c|c|c|c|}
\hline اسمه زن & برايمر رفت & برايمر بركشت & مقياس جورى بكالى & آنلينع & محصول \\
\hline CNTF & $\begin{array}{c}\text { CTTGCCTCTGTCTCCCTCTT } \\
\text { TG }\end{array}$ & $\begin{array}{l}\text { CTACTTGACGAAATATGC } \\
\text { CTGTGG }\end{array}$ & r & 9. & 114 \\
\hline BDNF & $\begin{array}{l}\text { AATGATGTGTCAAGTTGCT } \\
\text { TAGGC }\end{array}$ & $\begin{array}{l}\text { GAAATGGGATGGAGGCT } \\
\text { ATAAATGG }\end{array}$ & $r$ & $\Delta 9$ & 119 \\
\hline GDNF & $\begin{array}{c}\text { ACGCTTGGTGGTTGATTCT } \\
\text { GG }\end{array}$ & $\begin{array}{l}\text { GTTTCTGAGGGCACGAAG } \\
\text { GAG }\end{array}$ & r & 41 & ITF \\
\hline$\beta$-actin & $\begin{array}{l}\text { TGAAGATCAAGATCATTGC } \\
\text { TCCTC }\end{array}$ & $\begin{array}{l}\text { TCAGTAACAGTCCGCCTA } \\
\text { GAAG }\end{array}$ & $r$ & $\Delta \Lambda$ & iv. \\
\hline
\end{tabular}

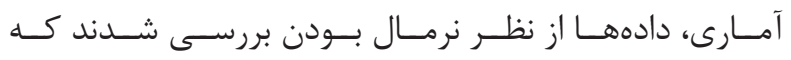

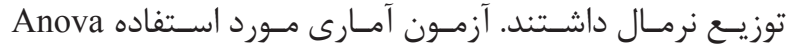

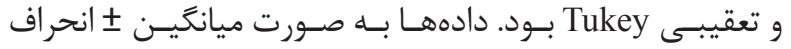

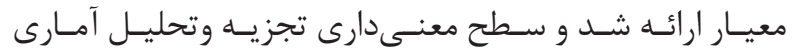

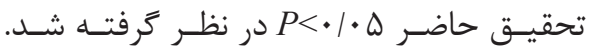

نافتهها

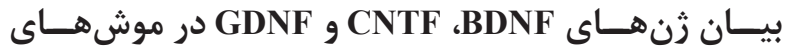

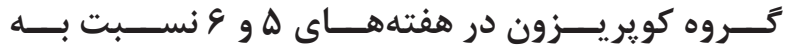

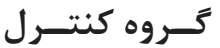

بيـان زنهــاى BDNF و GDNF در موشهــاى تحست

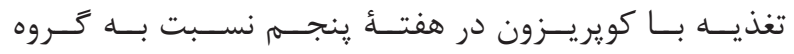

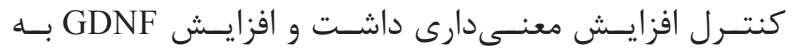

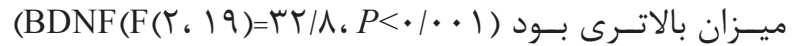

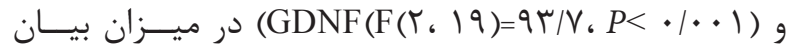

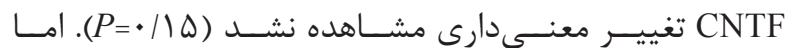

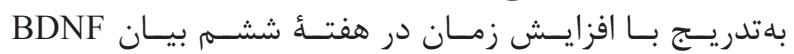

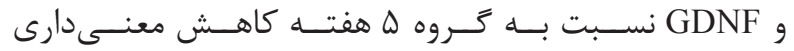

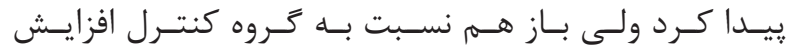

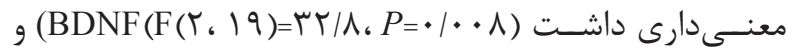

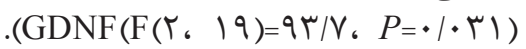

نتايج حاصل از رنََآميزى LFB و PAS

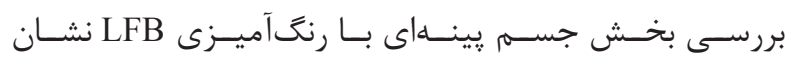

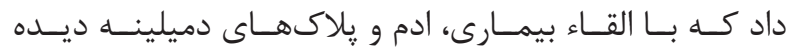

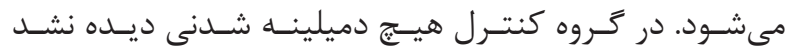

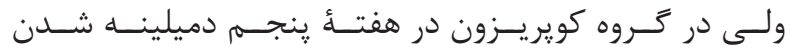

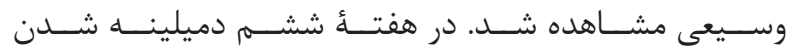

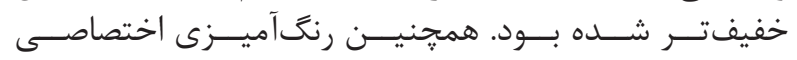

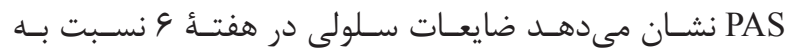

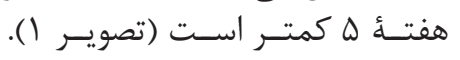

MOG نتايج حاصل از بررسى ايمنوهيستوشيمى

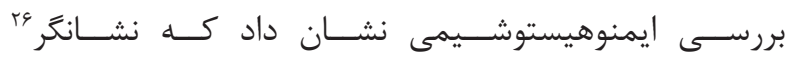

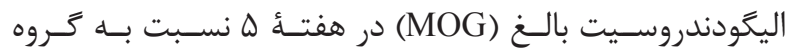

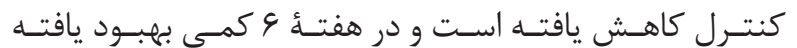

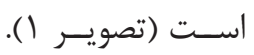

${ }^{20}$ Scale optical density; scale OD

${ }^{21}$ Optimum cutting temperature

${ }^{22}$ Luxol fast blue

${ }^{23}$ Periodic-acid schiff

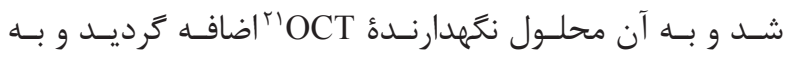

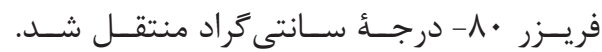

ســـس برشهــاى ه ميكرومتــر توســط دسـتخاه Cryostat در

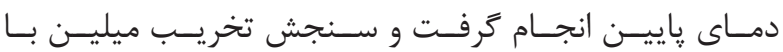

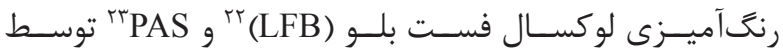

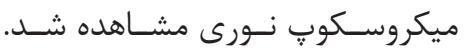
MOG بررسى ايمنوهيستوشيمى

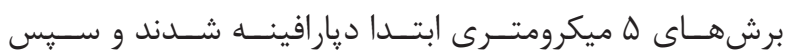

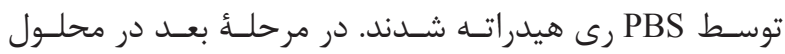

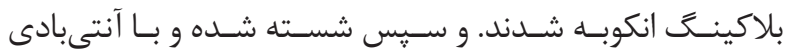

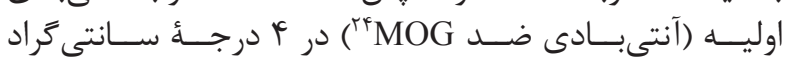

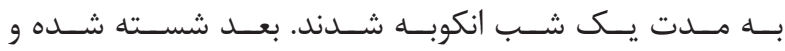

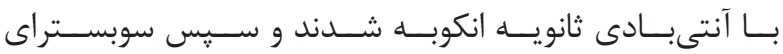

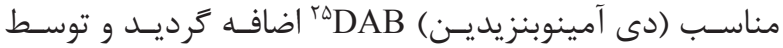

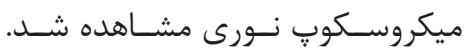
تجزيه و تحليل دادهها

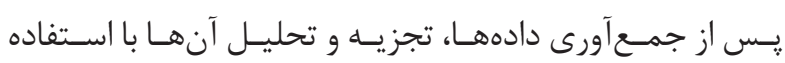

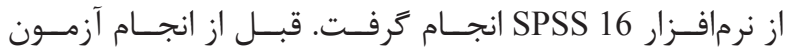

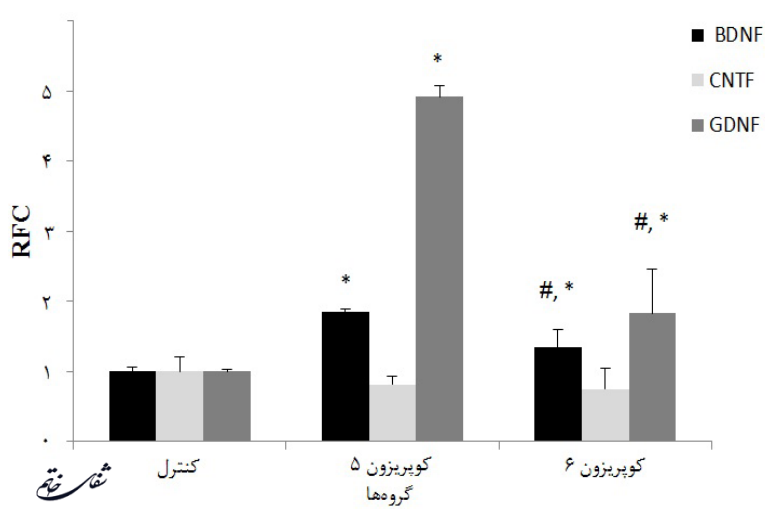

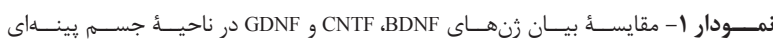

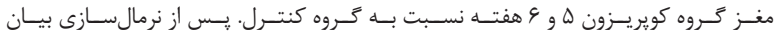

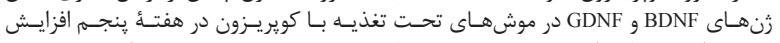

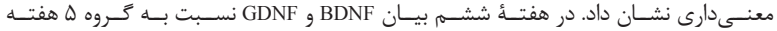

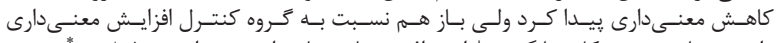

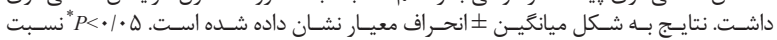

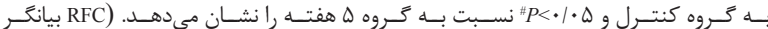
Relative Fold Changes

${ }^{24}$ Myelin-oligodendrocyte glycoprotein

25 3,3'-Diaminobenzidine

${ }^{26}$ Marker 


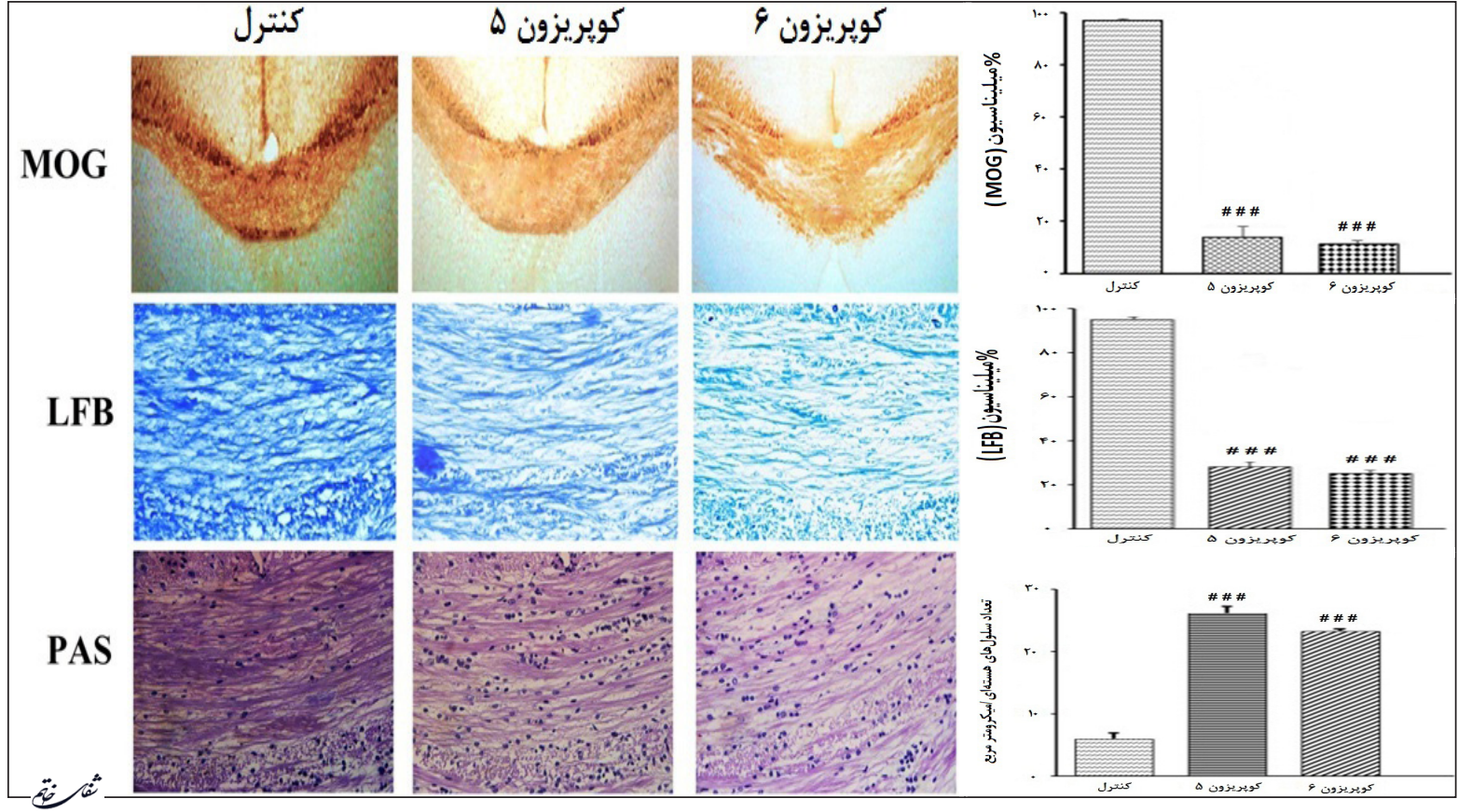

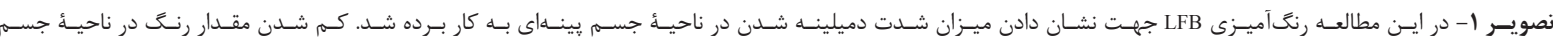

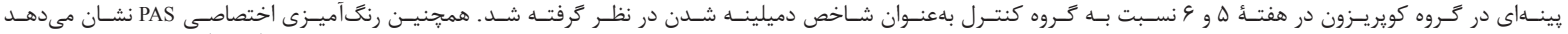

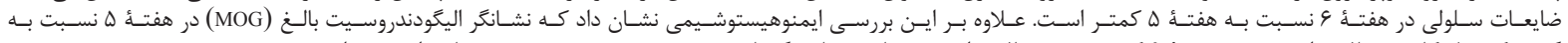

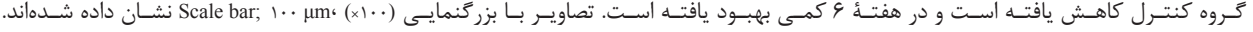

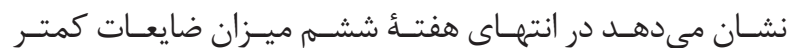

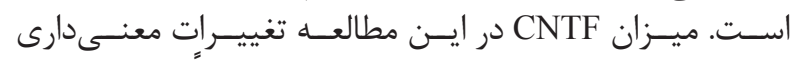

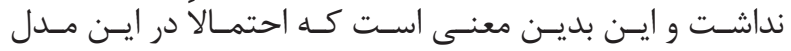

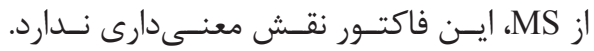

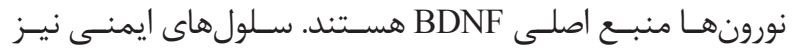

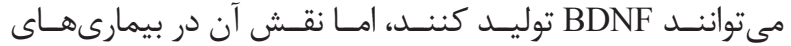

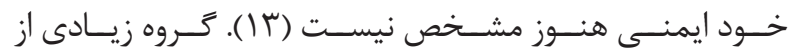

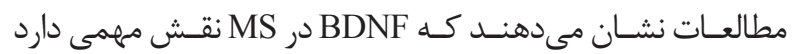

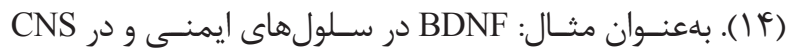

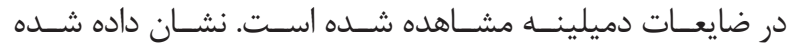

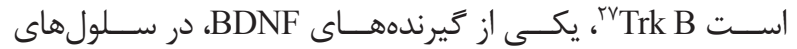

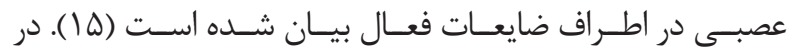

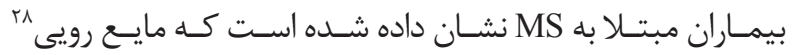

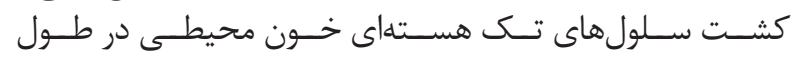

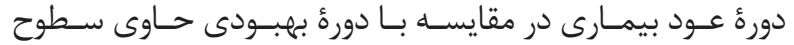

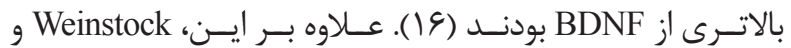

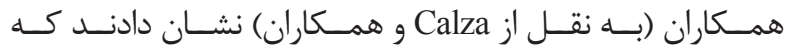

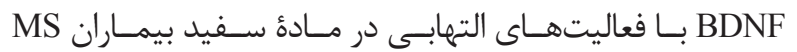

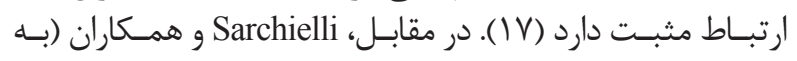

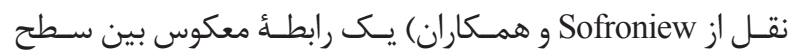

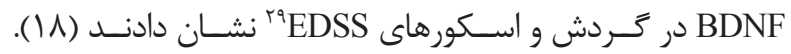

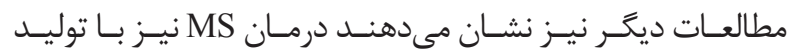

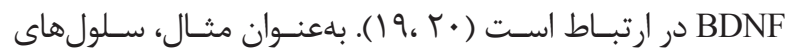

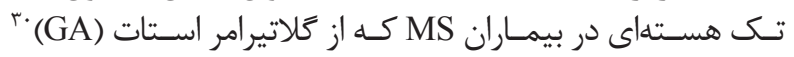

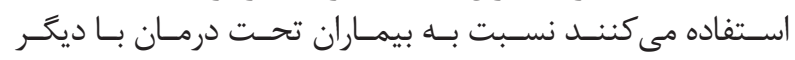

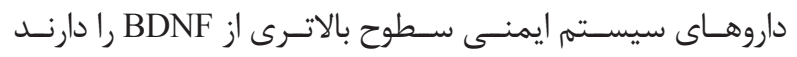

${ }^{27}$ Tropomyosin receptor kinase B

${ }^{28}$ Supernatant
بحث و نتيجه كيرى

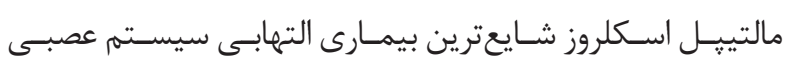

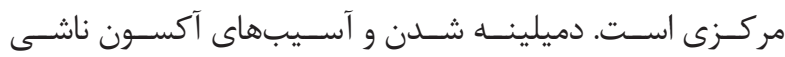

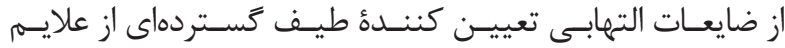

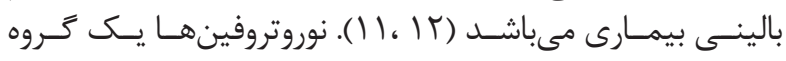

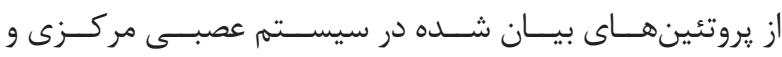

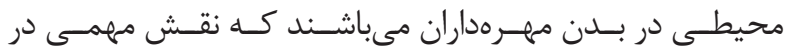

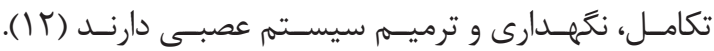

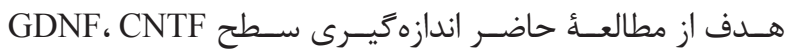

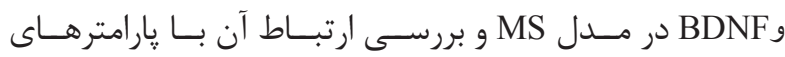

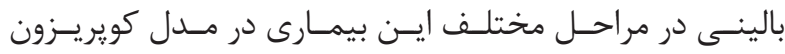

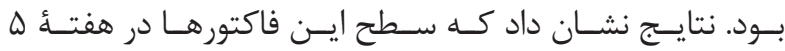

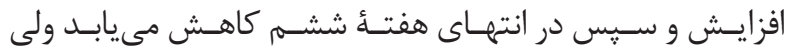

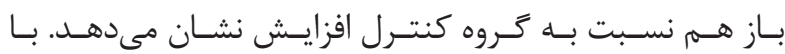

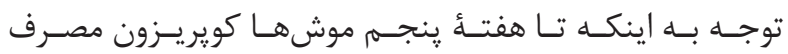

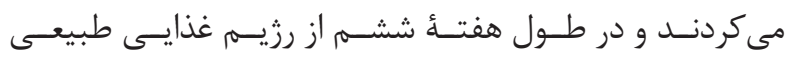

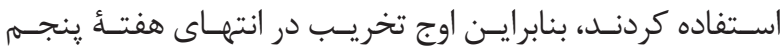

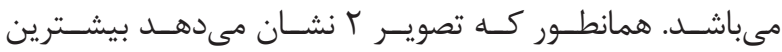

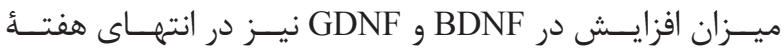

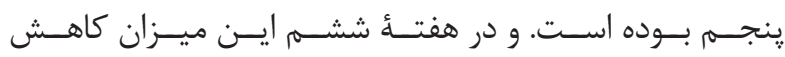

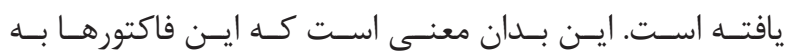

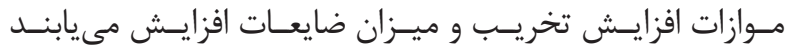

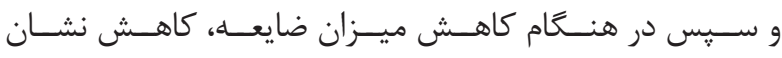

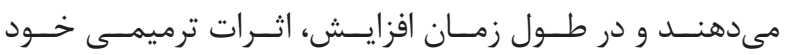

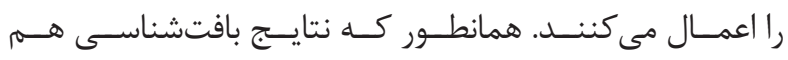

${ }^{29}$ Expanded disability status scale

${ }^{30}$ Glatiramer acetate 


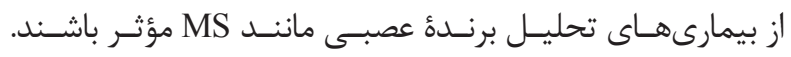

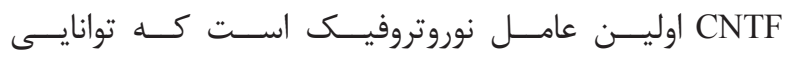

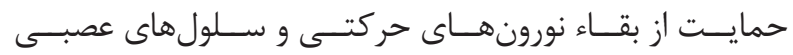

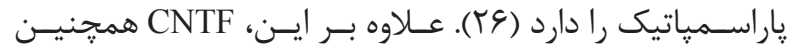

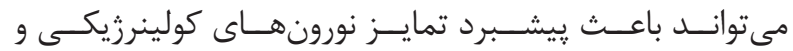

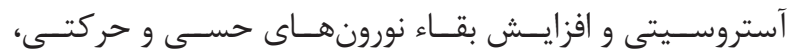

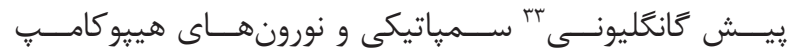

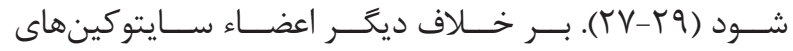

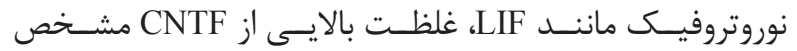

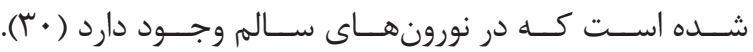

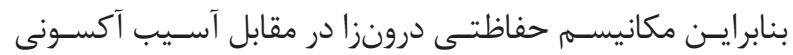

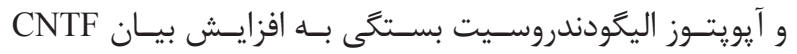

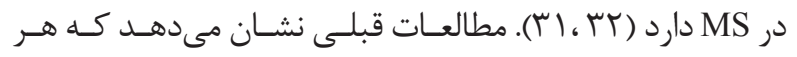

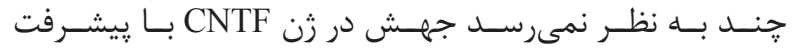

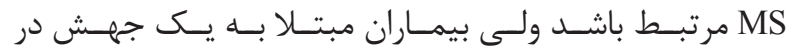

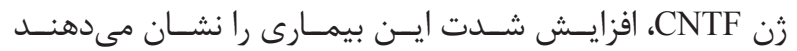

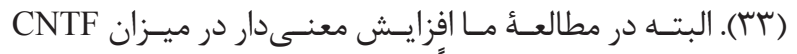

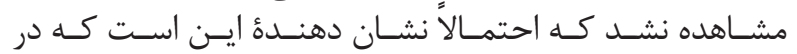

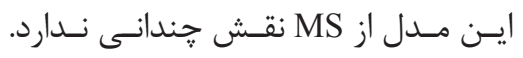

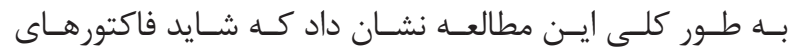

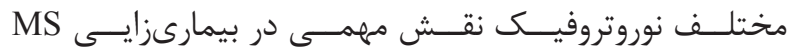

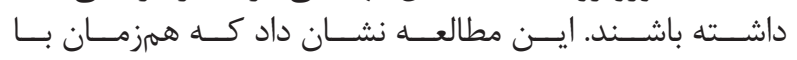

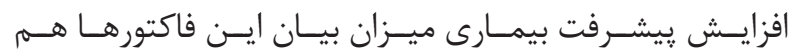

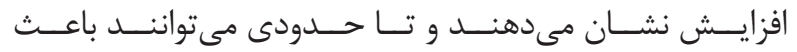

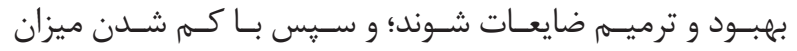

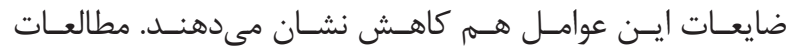

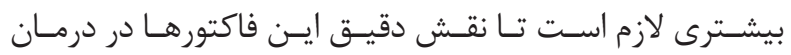

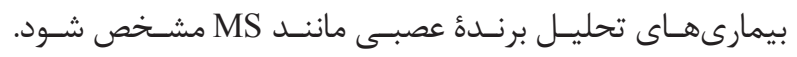

1. Frohman EM, Racke MK, Raine CS. Multiple Sclerosis--the plaque and its pathogenesis. N Engl J Med. 2006; 354(9): 942-55.

2. Polman CH, O’Connor PW, Havrdova E, Hutchinson M, Kappos L, Miller DH, et al. A randomized, placebocontrolled trial of natalizumab for relapsing Multiple Sclerosis. N Engl J Med. 2006; 354(9): 899-910.

3. Olson JK, Croxford JL, Calenoff MA, Dal Canto MC, Miller SD. A virus-induced molecular mimicry model of Multiple Sclerosis. J Clin Invest. 2001; 108(2): 311-8.

4. Stankoff B, Aigrot MS, Noël F, Wattilliaux A, Zalc B, Lubetzki C. Ciliary neurotrophic factor (CNTF) enhances myelin formation: a novel role for CNTF and CNTFrelated molecules. J Neurosci. 2002; 22(21): 9221-7.

5. Weihui Huang, Yadan Li, Yufeng Lin, Xue Ye, Dawei

${ }^{31}$ Neurturin

${ }^{32}$ Amyotrophic lateral sclerosis

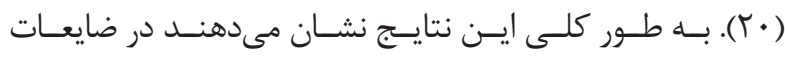

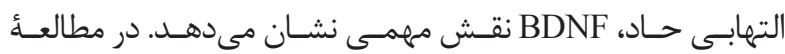

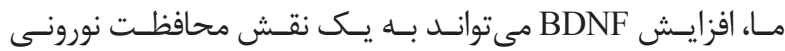

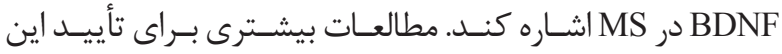
فرضيـه لازم اسـت

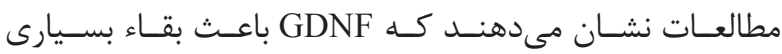

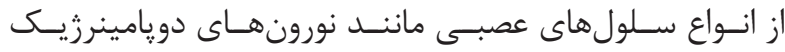

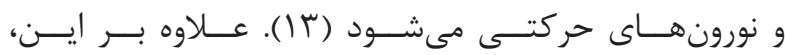
GDNF

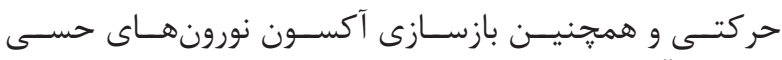

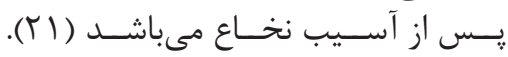
م BDNF

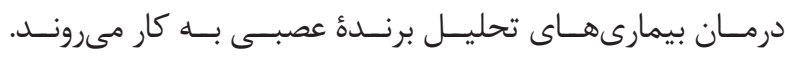

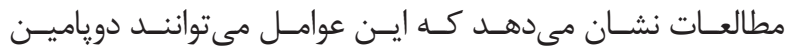

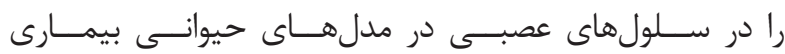

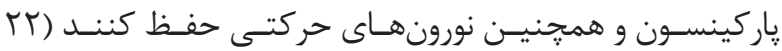

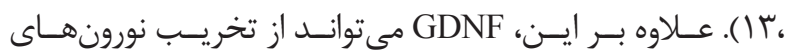

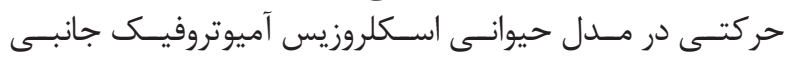
(ALS)

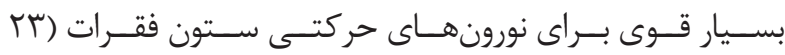

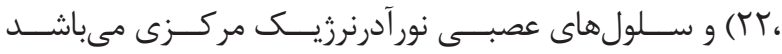

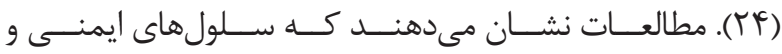

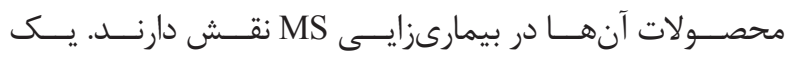

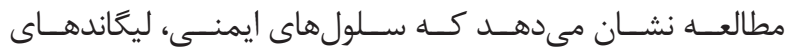

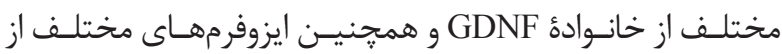

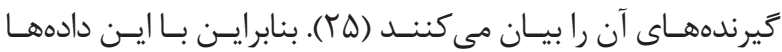

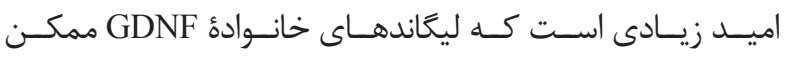

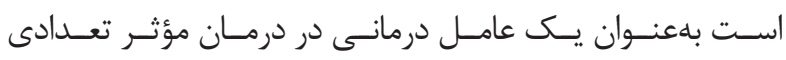

منابع

Zang. Effects of leukemia inhibitory factor and basic fibroblast growth factor on free radicals and endogenous stem cell proliferation in a mouse model of cerebral infarction. Neural Regen Res. 2012; 7(19): 1469-74.

6. Fang M, He D, Zhang F, Hu Z, Yang J, Jiang H. Antineuroinflammatory and neurotrophic effects of CNTF and C16 peptide in an acute experimental autoimmune encephalomyelitis rat model. Front Neuroanat. 2013; 7: 44. doi: 10.3389/fnana.2013.00044.

7. Caggiula M, Batocchi AP, Frisullo G, Angelucci F, Patanella AK, Sancricca C, et al. Neurotrophic factorsand clinical recovery in relapsing-remitting Multiple Sclerosis. Scand J Immunol. 2005; 62(2): 176-82.

8. Costello F, Stüve O, Weber MS, Zamvil SS, Frohman E. Combination therapies for Multiple Sclerosis: scientific rationale, clinical trials, and clinical practice.

${ }^{33}$ Preganglionic 
Curr Opin Neurol. 2007; 20(3): 281-5.

9. Blakemore WF, Franklin RJ. Remyelination in experimental models of toxin-induced demyelination. Curr Top Microbiol Immunol. 2008; 318: 193-212.

10. Livak KJ, Schmittgen TD. Analysis of relative gene expression data using real-time quantitative PCR and the 2(-delta delta C (T)) method. Methods. 2001; 25(4): 402-8.

11. Ebers G. Natural history of Multiple Sclerosis. J Neurol Neurosurg Psychiatry. 2001; 71(2): 16-9.

12. Frota ER, Rodrigues DH, Donadi EA, Brum DG, Maciel DR, Teixeira AL. Increased plasma levels of brain derived neurotrophic factor (BDNF) after Multiple Sclerosis relapse. Neurosci Lett. 2009; 460(2): 130-2.

13. Razavi S, Nazem G, Mardani M, Esfandiari E, Salehi H, Esfahani SH. Neurotrophic factors and their effects in the treatment of Multiple Sclerosis. Adv Biomed Res. 2015; 4: 53. doi: 10.4103/2277-9175.151570.

14. Götz R, Köster R, Winkler C, Raulf F, Lottspeich F, Schartl $M$, et al. Neurotrophin-6 is a new member of the nerve growth factor family. Nature. 1994; 372(6503): 266-9.

15. Urschel BA, Hulsebosch CE. Hulsebosch, Schwann cell-neuronal interactions in the rat involve nerve growth factor. J Comp Neurol. 1990; 296(1): 114-22.

16. Hardy K, Spanos S. Growth factor expression and function in the human and mouse preimplantation embryo. J Endocrinol, J Endocrinol. 2002; 172(2): 221-36.

17. Calzà L, Giardino L, Pozza M, Micera A, Aloe L. Time-course changes of nerve growth factor, corticotropinreleasing hormone, and nitric oxide synthase isoforms and their possible role in the development of inflammatory response in experimental allergic encephalomyelitis. Proc Natl Acad Sci U S A. 1997; 94(7): 3368-73.

18. Sofroniew MV, Howe CL, Mobley WC. Nerve growth factor signaling, neuroprotection, and neural repair. Annu Rev Neurosci. 2001; 24: 1217-81.

19. Shooter EM. Early days of the nerve growth factor proteins. Annu Rev Neurosci. 2001; 24: 601-29.

20. Althaus HH, Klöppner S, Schmidt-Schultz T, Schwartz P. Nerve growth factor induces proliferation and enhances fiber regeneration in oligodendrocytes isolated from adult pig brain. Neurosci Lett. 1992; 135(2): 219-23.

21. Ramer MS, Priestley JV, McMahon SB. Priestley, and S.B. McMahon, Functional regeneration of sensory axone into the adult spinal cord. Nature. 2000; 403(6767): 312-6.

22. Gash DM, Zhang Z, Ai Y, Grondin R, Coffey R, Gerhardt GA. Trophic factor distribution predicts functional recovery in Parkinsonian monkeys. Ann Neurol. 2005; 58(2): 224-33.

23. Henderson CE, Phillips HS, Pollock RA, Davies AM, Lemeulle C, Armanini M, et al. GDNF: a potent survival factor for motoneurons present in peripheral nerve and muscle. Science. 1994; 266(5187): 1062-4.

24. Arenas E, Trupp M, Akerud P, Ibáñez CF. GDNF prevents degeneration and promotes the phenotype of brain noradrenergic neurons in vivo. Neuron. 1995; 15(6): 1465-73.

25. Vargas-Leal V, Bruno R, Derfuss T, Krumbholz M, Hohlfeld R, Meinl E. Expression and function of glial cell line-derived neurotrophic factor family ligands and their receptors on human immune cells. J Immunol. 2005; 175(4): 2301-8.

26. Lin LF, Mismer D, Lile JD, Armes LG, Butler ET 3rd, Vannice JL, et al. Purification, cloning, and expression of ciliary neurotrophic factor (Cntf). Science. 1989; 246(4933): 1023-5.

27. Ernsberger U, Sendtner M, Rohrer H. Proliferation and differentiation of embryonic chick sympathetic neurons: effects of ciliary neurotrophic factor. Neuron. 1989; 2(3): 1275-84.

28. Saadat S, Sendtner M, Rohrer H. Rohrer, ciliary neurotrophic factor induces cholinergic differentiation of rat sympathetic neurons in culture. J Cell Biol. 1989; 108(5): 1807-16.

29. Lillien LE, Sendtner M, Rohrer H, Hughes SM, Raff MC. Type-2 astrocyte development in rat-brain cultures is initiated by a cntf-like protein produced by type-1 astrocytes. Neuron. 1988; 1(6): 485-94.

30. Stöckli KA, Lottspeich F, Sendtner M, Masiakowski P, Carroll P, Götz R, et al. Molecular cloning, expression and regional distribution of rat ciliary neurotrophic factor. Nature. 1989; 342(6252): 920-3.

31. Schönrock LM, Gawlowski G, Brück W. Interleukin-6 expression in human Multiple Sclerosis lesions. Neurosci Lett. 2000; 294(1): 45-8.

32. Vanderlocht J, Hellings N, Hendriks JJ, Vandenabeele F, Moreels M, Buntinx M, et al. Leukemia inhibitory factor is produced by myelin-reactive T cells from Multiple Sclerosis patients and protects against tumor necrosis factor-alpha-induced oligodendrocyte apoptosis. J Neurosci Res. 2006; 83(5): 763-74.

33. Giess R, Mäurer M, Linker R, Gold R, WarmuthMetz M, Toyka KV, et al. Association of a null mutation in the CNTF gene with early onset of Multiple Sclerosis. Arch Neurol. 2002; 59(3): 407-9. 